\title{
NATO AND SWEDEN JOINT LIVE EXPERIMENT ON NEC
}

\author{
Kristell ARNELL
}

\begin{abstract}
The overall objective for the project was to test if a "service oriented architecture" could facilitate the vision in the NATO NEC Feasibility Study, that states "Network Enabled Capability (NNEC) involves the linking together of sensors, decision makers, and weapon systems, as well as multinational military, governmental, and non-governmental agencies in a seamless, collaborative, planning, assessment and execution environment." The idea was to see if the utilization of NATO NEC developments together with Swedish NDB results could bring about new techniques that were more flexible and dynamic in its support of civil military co-operation and the Comprehensive Approach. The result of the live experiment was overwhelming, in about some six month the project team succeeded to federate together some 30 different types of information and C2 systems were both civil and military units could share the same operational picture and retrieve it from their own specialized systems. The federated system consisted of fully blown operational systems as the NATO AWAC to system on the experimental level. A main contributor to the success was the utilization of a Service Oriented Architecture. To verify the operational side of NEC, four different vignettes were created under the umbrella of the protection of a high level meeting in the Swedish city of Göteborg. Operating together in the scenarios was several different NATO entities, Swedish military units, the Swedish Police, the Swedish Rescue Service, Swedish SOS Alarm (911/112 functionality) and the Swedish Civil Contingencies Agency. The federated information sharing environment was accredited by both Swedish and NATO Security Authorities.
\end{abstract}

Keywords: Comprehensive Approach, NATO, Network Enabled Capability, C2 capabilities, Defence Support to Civilian Authorities.

\section{Introduction}

The project was conducted as a collaborative effort between SwAF, FMV, MSB (Swedish Civil Contingencies Agency) and NC3A with the goal of demonstrating what NNEC/NBD (Swedish equivalent of NEC) could achieve in terms of mission effectiveness, interoperability, flexibility etc. This would be achieved by executing a number of live vignettes from a scenario with an operational context that highlights 
the different aspects of NBD/NNEC. A major challenge for the project was to undertake all the required work in a fairly short time-frame for such a large endeavour. The approval and foundation phase of the project was conducted in 2007 and the majority of the project activities, including the demonstration event, were conducted in the first 9 months of 2008.

The overall objective for the project was to test if a "service oriented architecture" could facilitate the vision in the NATO NEC Feasibility Study, that states "Network Enabled Capability (NNEC) involves the linking together of sensors, decision makers, and weapon systems, as well as multinational military, governmental, and nongovernmental agencies in a seamless, collaborative, planning, assessment and execution environment.”

The project was essentially a technical project with the aim of demonstrating the foundations of NNEC/NBD techniques and architectures and their application to a range of military and civilian situations.

To add credibility to the demonstration and to more effectively support current NNEC/NBD development it was necessary to demonstrate systems and operators working together in a live operational environment. To support the live demonstration of NEC concepts and technologies, an operational scenario was created. The scenario was the protection of a fictitious high profile event in the city of Göteborg, Sweden. Several vignettes from the scenario were developed that were designed to emphasise a particular aspect of NEC concepts and technologies during the demonstration. To be able to demonstrate the different aspects of NNEC/NBD concepts and technologies, operational and experimental systems manned by operators from respective organizations were federated.

In the initial phase of the project we imagined the possibilities that would emerge if it was feasible to assemble and federate various systems for which there were originally no plans that they should ever be employed collectively or collaboratively such as our Swedish/NATO joint protection scenario. In this scenario:

- A Swedish police unit accessed information from a NATO ISR system from the MAJIIC project;

- A Swedish police unit accessed information from a NATO ISR system from the MAJIIC project;

- An operator on a NATO system accessed sensor information from Swedish civilian Air and Sea data providers; 
- $\quad$ The Swedish Air picture was combined with Air tracks from a NATO Air surveillance system;

- A civilian organization accessed military produced and exploited information;

- All participants shared the same "Common Operational Picture” enabling improved collaboration;

- The AWACS track service published to the mission service layer was discovered and connected as a new sensor to the Swedish COP service and other consumers in the enterprise including a Swedish Police command vehicle.

The inherent flexibility and agility of the Service Oriented Architecture approach was demonstrated by the rapid reconfiguration of the demonstration architecture using the "SitSyst" orchestration tool. By utilizing the NNEC/NBD principles we demonstrated that it is indeed possible to assemble services from systems in accordance with the needs of a requester. This in turn enables better support to the operational demands affording the ability to swiftly put together organizations for future crisis in different domains that were not originally foreseen.

It was demonstrated that ad hoc updates of capabilities as provided by military and civilian organizations during joint missions as driven by operational demand is feasible. This is in contrast to where updates of capabilities could only be performed months prior to the mission.

\section{Architecture/Technology}

The experiment and demonstration had as its primary focus three main areas:

Flexibility - illustrating technical (system development and operation) and operational flexibility, where Service Oriented Architecture (SOA) and the service concept are key enablers to achieve flexibility.

Interoperability - highlighting technical interoperability between military and civilian partners and organizations, and across national boundaries.

Realization - to show a possible "Quick Win" that may be fully realized within a very short timeframe (1-2 years).

To reduce risks and for the sake of efficiency, a number of systems and components previously used in NEC experiments and demonstrations - both by Sweden and NC3A - were included and modified for the demonstration. These included, from NATO, some of the NNEC core services (see below) as well as the MAJIIC Coalition Shared Data (CSD) server and the Networked Interoperable Real-time Information 
Services (NIRIS), the Kongsberg Exploitation System (KES) and the Norwegian Collection Coordination and Information Management System (NORCCIRM). From Sweden, the re-used components included service bridges to sensor systems such as AIS, MSSR, PS740 and ground sensor net and selected components from the Swedish NBD experiment platforms (core services and COP services).

There were five primary technical aspects that were worked on by the technical team, which included:

- $\quad$ Real-time track exchange services, such as tracks and video;

- Near Real-time ISR Services, such as planning, imagery, reports and exploitation;

- Collaboration services, such as chat and VOIP;

- Emergency and First responder services;

- System management, discovery and security standards for a SOA architecture.

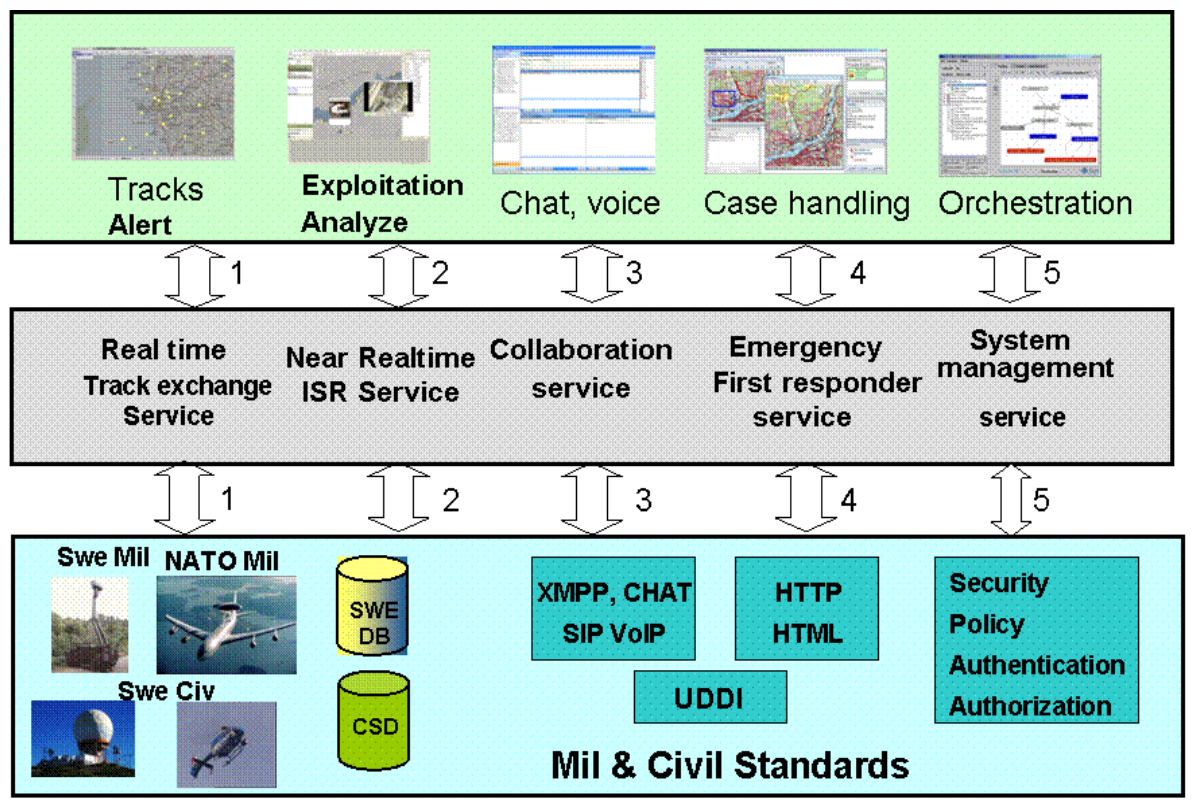

Figure 1: Technical focus area. 


\section{Frameworks and reference models}

Architecture frameworks used

The experiment used existing architecture frameworks from NATO (the NATO Architecture Framework, or NAF3) and Sweden to produce a common Target Architecture document including the new views for service-orientation (NSOV). During this work a lot of experience from the Swedish NBD development with architecture frameworks was also utilized.

\section{Technical Reference Models used}

During the architecture and design work both Swedish NBD and NATO NEC technical reference models (TRM) were used. Technical reference models are important for engineering purposes to support structure and consistency in architecture and design, to enhance communication and understanding between people, and to support technology comparisons.

This convergence of the NNEC and NBD TRMs made our joint architecture and design work easier and faster. The overall structure and the details are well aligned between NNEC and the Swedish NBD/FMLS.

\section{Other Major References/Design Guidance}

An important trend in the IT industry has been the movement towards open standards. These are not industry groups producing proprietary protocols that are restricted in their application, but public and transparent standards bodies that are dedicated to ensuring the interoperability of (for example) Web services applications.

Two of the primary standards bodies in the SOA world are the World Wide Web Consortium (W3C) and the Organization for the Advancement of Structured Information Standards (OASIS). Both the Swedish military and NC3A are being careful to develop, in particular, their Core Enterprise Services (the elements that underpin and enable the SOA environment) following the most recognized and common standards from these bodies. This consistency with the industry-leading open standards helped smooth the way for the collaboration.

\section{Technical Principles}

NEC Integration levels

To be able to provide and consume NEC services from existing systems they need to be integrated and adapted to:

- Make information from the legacy system available on the network; 
Swedish NBD TRM

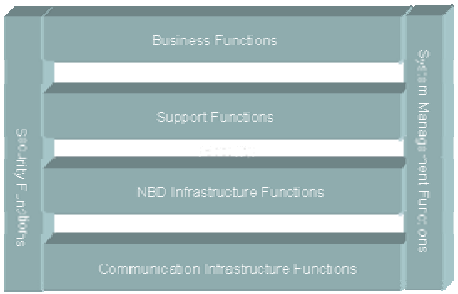

NATO NEC TRM

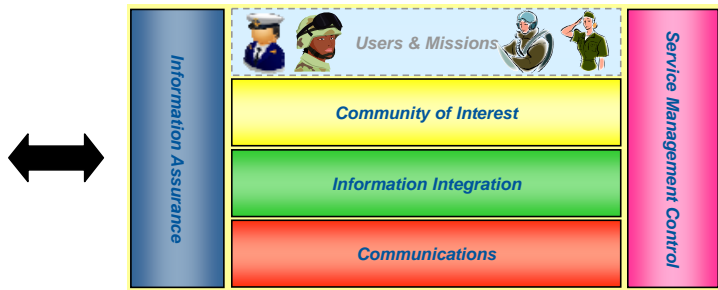

Figure 2: Comparison between Swedish NBD TRM and NATO NEC TRM.

- Allow remote control of the legacy system and/or equipment connected to the legacy system;

- Make specific services from the network and information provided by those services available to the legacy system and/or to operators in the system.

This integration may be performed to different levels for different services.

The experiment re-used existing design rules, integration patterns and system integration experiences from the Swedish NBD program (e.g., DR Legacy integration with five different integration levels, a number of integrated legacy systems) and NNEC.

These integration patterns are also valid for some COTS and open-source systems.

Conclusion/Lessons learned: the use of different integration levels for legacy systems enables a step-wise deeper and wider NEC integration of existing systems:

- $\quad$ The legacy system and the network adaptation can have separate lifecycles;

- The necessary modifications of the actual legacy system can be minimized.

\section{Bridging}

Bridging was used for a number of services as the implementation of "bridges" to existing systems was seen as quick and low-cost interoperability enablers. Several such bridges were implemented.

Track exchange from service bridge:

- $\quad$ NIRIS in NATO system Link-16-messages;

- $\quad$ SWE track bridge for AIS (automatic identification system), MSSR (multiple secondary surveillance radar), PS 740 (sea radar), ground sensor fusion node; 
- $\quad$ SWE civil track bridge from BFT (truck and CBRN measure system).

ISR Information discovery:

- $\quad$ SE federated search in SE metadata repo and NATO CSD;

- $\quad$ NATO replication of SE metadata and ISR products into CSD.

Conclusion/Lessons learned from: The use of existing bridges from the NATO side and the Swedish side made the integration work easier and faster $=>$ realization (Quick Win) Bridges is an important component for integrations of Legacy and to be interoperable to coalition partner:

In SWE NBD description of service bridges there are a couple of principals to take care of how complex you want the bridge to be (one or multiple services).

- Security level and functions internal organization or between different organizations (cross-domain);

- Service management functions;

- Bridges to legacy depending on Safety, real-time properties, information volume.

\section{Cross-domain, cross-platform interoperability of web services}

The supporting security libraries need to be implemented with necessary language and operating system combinations. In the experiment setup there was a mix of different operating systems and web service containers (Linux/Apache Tomcat, Windows/IIS).

A number of lessons were identified following the demonstration in this security experiment:

- $\quad$ Sweden needed to implement support for SAML-tokens in their Java/web service framework, NATO security lib was based on C\# and bridge towards Sweden metadata repository was implemented in Java. Therefore, the integration was not only a matter of plug-and-play, rather there has to be coordination to achieve sufficient functionality.

- There is also a not negligible amount of work involved in certificate management and construction of a correct SAML token, e.g., to ensure that all attributes with correct values are used.

\section{Conclusions}

An important conclusion from our experiment is that it is indeed feasible to adjust and adapt today's technical systems (legacy systems) to a service oriented environ- 
ment. What seem to be lacking are common procedures and methods on how to apply the new techniques regarding information sharing in both military and civilian domains.

Politicians and leaders of different organizations will continue to have very high demands that investments made on different systems should not be obsolete just because they could not foresee all the future environments and situations that they would be utilized in.

The SWEDEN - NATO Joint Live Experiment on NEC project has clearly demonstrated that it is possible to use operational systems and integrate them in a new way (Federation of services instead of point-to-point life cycle integration of the various systems ) and in doing that achieve new information sharing abilities. Systems can be federated to meet operational demands not known until fairly late before the operation is conducted. At the same time the individual systems remain autonomous, meaning that each organization can control that only agreed information is shared. A user should be able to work in his or her own system and still be able to share information with others on an agreed level.

NNEC/NBD relies on a Service Oriented Architecture to achieve its prophecy. Service Oriented Architectures can be realized using multiple standards from civilian standardization organizations which in some cases are overlapping, i.e. convergence efforts as demonstrated within this project are necessary to achieve interoperability.

NNEC has been inspired by ongoing NEC related efforts within the various nations including the NEC efforts in Sweden. So it is no surprise that the Swedish NBD technical architecture and NNEC Networking and Information Infrastructure have many architectural commonalities. Both rely heavily on standards from civilian standardization organizations as well as standards from NATO. However, standards may overlap and they may be employed in various ways which are not necessarily compliant. Thus, experimentation is crucial for ensuring uniform utilization of a consistent set of specifications. Furthermore, as a principle, in order to enable interoperability between civilian and military organizations, standards from civilian standardization organizations should be prioritized when selecting the standards that underpin the NEC technical architectures.

The experiment has taught us that there are two groups of stakeholders that need to be addressed in order to be able to bring about a NEC/NBD environment; the operators who want to see operational benefits and also the procurement side of the organization who need to understand what they can gain from "service oriented approach" regarding procurement effectiveness. 
This experiment has demonstrated that by focusing on some areas of NBD/NNEC it is relatively easy to achieve concrete results. It is also possible to evolve NEC capabilities incrementally in smaller steps that are more easily brought to bear in the operational environment.

Projects like this need to invest time to reach a common understanding for what NNEC/NBD means and to derive the specific objects of the project.

One of the most important lessons learned from the project is that good team spirit along with collaborative, iterative work and support, and open information sharing improved the overall progress and significantly increased the success likelihood of the demonstration.

The relative few stakeholders that were represented in the project management team provided a foundation for rapid decision making which is essential to drive a project like this forward. That does not hamper the ability to bring in supplementary partners by the project legal partners.

For those interested in all the details from the project, including all services utilized, network design, target architecture etc, the jointly produced final report is recommended. It is a NATO unclassified document and named "NATO and Sweden joint live experiment on NEC: A first step towards a NEC realization."1

\section{Notes:}

${ }^{1}$ Kristell Arnell, et al., NATO and Sweden Joint Live Experiment on NEC: A First Step Towards a NEC Realization, Reference Document DOP-D125-09 (The Hague: NATO Consultation, Command and Control Agency, March 2009), Available at www.centriclabs.se/files/pdf/SWE-NATO_informationsdelningsexperiment-slutrapport.pdf. 


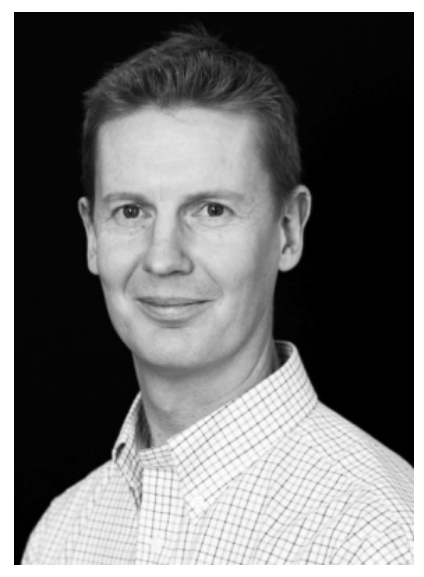

Lt Col (ret.) KRISTER ARNELL was born in Rättvik, Sweden, on $6^{\text {th }}$ of February in 1964. In 1984 he started his military career. After graduating from the Air Force academy in 1987, in the fighter control branch. The same year he was stationed as an air surveillance officer at the Jämtland Air force Wing in the middle of Sweden. Soon after he started his specialization as weapons controller, fighter allocator and master controller. In 2003 he assumed position as head of the planning section for the fighter controlling branch at the Swedish Air Force staff. In 2005 he graduated from the Swedish National Defence College with honours. Soon after he assumed the position as the Swedish Armed Forces program manager for the Swedish NBD (NEC) development program. 2007 he was appointed Battalion Commander for the Swedish Air Force Command and Control Battalion. During 2007 and 2008 he was also the Swedish project manager for the SWE/NATO Live experiment on NEC. He retired in February 2009 and is now working as a senior management consultant at Centric Labs AB (SWE). Krister is married to Anja and they have two sons - Agaton and Lukas. 